\title{
Structure of a disk-like mesophase
}

\author{
A. M. Levelut \\ Laboratoire de Physique des Solides (*), Université Paris-Sud, 91405 Orsay, France
}

(Reçu le 7 novembre 1978, révisé le 18 décembre, accepté le 21 décembre 1978)

\begin{abstract}
Résumé. - Nous avons effectué des diagrammes de diffraction des rayons $\mathrm{X}$ de la phase mésomorphe des deux dérivés hexasubstitués du terphénylène. Nous confirmons la structure déjà proposée pour la phase mésomorphe d'une molécule analogue : les molécules forment des colonnes régulièrement disposées aux nœuds d'un réseau hexagonal. En outre, il est possible de décrire plus précisément l'arrangement des groupes terphényl à l'intérieur d'une colonne.
\end{abstract}

Abstract. - We have obtained X-ray diffraction patterns from the mesomorphic phase of two derivatives of terphenylene. The observations agree with the structure already proposed for the mesomorphic phase of a similar molecule : the molecules are stacked in columns and the columns form a regular hexagonal array. In addition it is possible to give a more accurate description of the positions of the terphenyl groups in a column.

Evidence for thermotropic mesomorphism in a pure system of disk-like molecules has been reported by S. Chandrasekhar et al. [1]. These authors have investigated the benzene-hexa-n-alkanoates and they have proposed a structure based on X-ray data : the disks are stacked in columns and are irregularly spaced the columns forming a regular hexagonal array. This structure is similar to that of the hexagonal phase obtained for soaps and other lyotropic systems [2].

At the same time, other disk-like molecules were synthesized which exhibit a mesomorphic state $[3,4]$. Thus, it was interesting to perform X-ray experiments on such a system and to investigate if the model proposed by Chandrasekhar is characteristic of a new class of mesomorphic compounds.

1. Experiments. - We have studied two hexaalcoxy derivatives of the triphenylene :

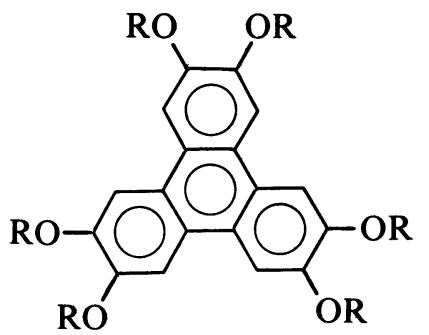

$$
\begin{aligned}
& \mathrm{R}=\mathrm{C}_{5} \mathrm{H}_{11} \\
& \mathrm{R}=\mathrm{C}_{7} \mathrm{H}_{15}
\end{aligned}
$$

The $\mathrm{C}_{5}$ derivative exhibits a mesomorphic phase between $69^{\circ}$ and $122^{\circ} \mathrm{C}$ while the $\mathrm{C}_{7}$ derivative is

(*) Laboratoire associé au C.N.R.S. mesomorphic between 65 and $95^{\circ} \mathrm{C}$ [3]. The samples were held in a Lindeman glass capillary, the temperature of which was constant within $0.5^{\circ} \mathrm{C}$. The $\mathrm{X}$-ray beam came from a double bent graphite monochromator through a collimator of $0.5 \mathrm{~mm}$ and we used $\mathrm{CuK}_{\alpha}$ radiation. The diffracted $\mathrm{X}$-rays were collected on a flat film.

It is relatively easy, at least for the pentyl compound, to obtain large oriented domains (the planes of molecules are all parallel) by waiting several hours just below the mesomorphic $\rightarrow$ isotropic transition. Such domains are stable for few days and thus we can obtain single domain patterns with various orientations and sample temperatures. (After a few days and many temperature cycles in the mesophase, a slight decomposition of the sample takes place due to the heat treatment.)

2. Experimental results. - The two characteristic patterns are shown in figures 1 and 2 and these patterns have been obtained with the pentyl derivative. In figure 1 , the incident $X$-ray beam is parallel to the molecular planes. The most striking feature consists of an intense diffuse line (a) lying in a direction parallel to the molecular plane. Such a line comes from diffracted X-rays with a scattering vector ending in a reciprocal sheet parallel to the molecular plane. Since a set of equidistant planes is the reciprocal image of a periodic line, we can conclude that the molecules are regularly stacked in parallel columns. The distance between two neighbouring reciprocal planes gives us 


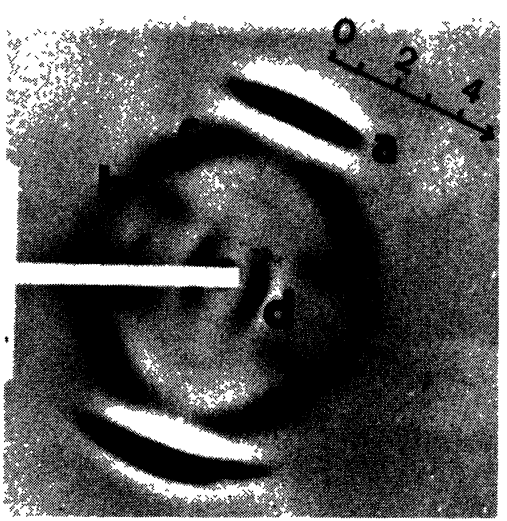

Fig. 1. - Diffraction pattern of a single domain of the mesophase of hexapentyloxy terphenylene. Incident beam parallel to the molecular planes : a) fundamental reciprocal plane, b) superlattice diffuse sheet, c) diffuse ring characteristic of the liquid-like order of the chains, d) diffuse sheets parallel to the sixfold axis, also visible in figure 2. The scale is drawn in reciprocal units of the hexagonal lattice.

the interspacing distance of the molecules in each column and this distance is $d=3.59 \AA$. The geometry of the apparatus is not suitable for the observation of the high level planes and we only see the first one. The scattered intensity in this plane seems to depend only on the molecular structure factor and thus the $z$ coordinates ( $o z$ is taken parallel to the axis of the columns) of molecules belonging to different columns are uncorrelated.

At low temperatures $\left(\simeq 80^{\circ}\right)$, a lighter scattered intensity appears in a parallel sheet closer to the origin of the reciprocal space (b). This feature is indicative of a superlattice periodicity in the direction of the stacks of molecules. The modulation of the diffracted intensity inside this sheet is characteristic of the diffraction pattern of a helix [5] (the intensity is zero along the $o z$ axis). The pitch of the helix is $D=13 \AA$. Besides the diffuse sheets, we can see a diffuse ring (c) corresponding to a mean reciprocal distance of $1 / 4.5 \AA^{-1}$. This ring thus seems characteristic of the liquid-like order between the aliphatic chains; this ring is strongly modulated at $85^{\circ} \mathrm{C}$, but by heating the sample, this modulation becomes weaker. Nevertheless the intensity is maximum at an angle of about $20^{\circ}$ to the axis of the stacks. Let us remark that a liquid-like order of the chains does not imply an isotropic distribution of the C-C links; the mean direction of the chain is probably slightly out of a plane perpendicular to the stack axis. At the same time, the superlattice diffuse sheet $(D=13 \AA)$ disappears at about $100^{\circ} \mathrm{C}$. Diffuse sheets parallel to the $z$ axis (d) are also visible, their origin will be discussed further when we consider the second kind of pattern.

Figure 2 corresponds to a sample in which the molecular planes are perpendicular to the X-ray beam. We thus obtain an image of the zero level reciprocal plane perpendicular to $o z$ and it is characteristic of the correlation between the in-plane oxy

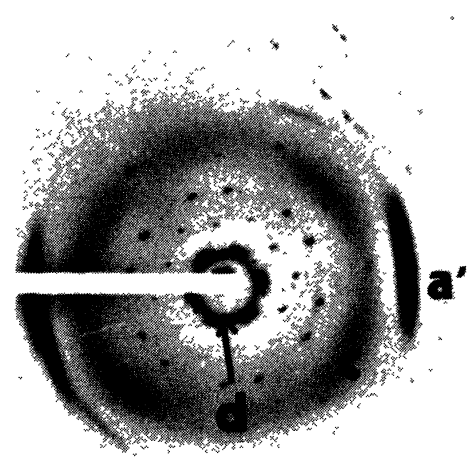

Fig. 2. - Diffraction pattern of the same single domain as in figure 1. The incident beam is perpendicular to the molecular planes. Other small domains are also in the X-ray beam and one can see fundamental diffuse sheets $\left(a^{\prime}\right)$ and extra Bragg spots coming from these domains $\left({ }^{1}\right)$.

coordinates of different molecules. First, we see sharp Bragg spots lying on a hexagonal two dimensional lattice ( $h k 0$ spots). As these spots lie only in the equatorial plane, they originate in a hexagonal $\left({ }^{2}\right)$ packing of rods of indefinite length : each rod is a stack of molecules. The distance between two rods is $a=18.94 \AA$. Besides the Bragg spots, a small part of the scattered intensity is localized on the rows [ $h k 0]$ of the two dimensional lattice and if we compare figure 1 and 2, these rows come from scattered intensity localized on diffuse sheets parallel to the $z$ axis and containing the $h k 0$ rows. In fact, they have a limited extension in the $z$ direction $\left(\left|S_{z}\right|<\frac{1}{15} \AA^{-1} S_{z}\right.$ being the $z$ projection of the scattering vector). We can only see the first set of planes $h= \pm 1, k= \pm 1$ or $h-k= \pm 1$ in figures 1 and 2 ; the intensity of other orders is weaker but they are visible on the original patterns. Similar diffuse planes have been observed on single domain patterns of SmB and $G$ phases [6]. In this last case, the diffuse scattering in plane is provided by longitudinal motions of strings of aligned molecules. In the case of disk-like molecules, they are not aligned along [ $h k 0]$ rows, so the diffuse scattering corresponds to longitudinal motions of bands of molecules $15 \AA$ wide in the $z$ direction. A description can also be given in terms of elastic modes [7] : the X-rays are scattered by bending

$\left({ }^{1}\right)$ The exposure times are the same for the two diffraction patterns. Nevertheless, it is difficult to obtain a good idea of the respective intensity of the different features since in spite of the very good photographic reproduction obtained by Mr Saint Martin of our laboratory, the contrasts are considerably damped through each stage of the replication.

$\left({ }^{2}\right)$ A hexagonal two-dimensional lattice implies either a threefold symmetry axis or a sixfold axis and we cannot distinguish between the two possibilities from our $\mathrm{X}$-rays observations. 
modes of columns of molecules. For a wave length $>15 \AA$, the frequency is very low and the intensity of the X-rays scattered by these modes becomes very high. Moreover they are uncoupled from stack to stack except in a direction parallel to the displacement (Fig. 3). Further, the diffuse ring at $S=\frac{1}{4.5} \AA^{-1}$ is still visible but with a weaker intensity compared to the pattern of figure 1 .

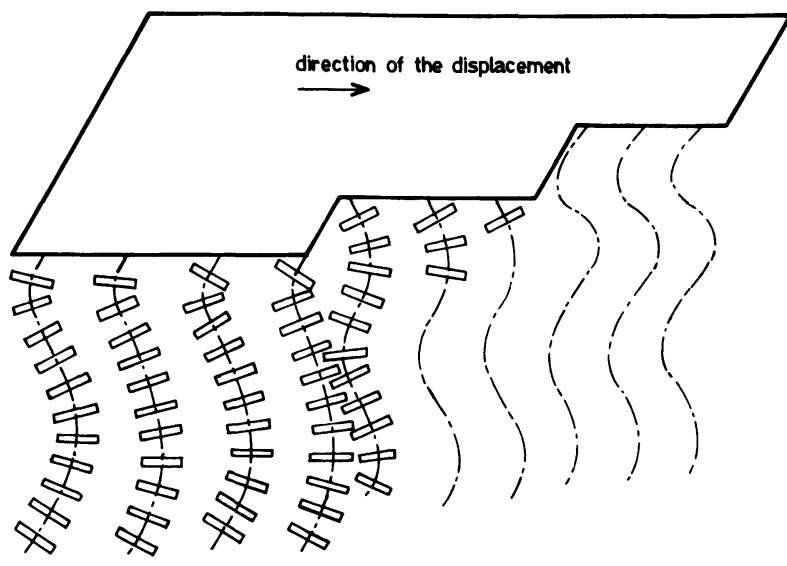

Fig. 3. - Schematic representation of the bending modes of the columns of molecules.

The last point we want to discuss about the single domain pattern of hexapentyloxy terphenylene concerns the relative intensity of the scattered X-rays in the different levels of the reciprocal space. We have to compare the zero level plane i.e. the hexagonal Bragg spots $h k 0$, the fundamental first level $(d=3.59 \AA)$ and the superlattice first level $D=13 \AA$.

Let us consider a stack of $d$ equally spaced molecules, the molecules form a helix of pitch $D$, if $D / d$ is irrational, the diffracted intensity is mainly localized on two kinds of reciprocal planes [5]; fundamental planes with $S_{z}=n / d$ and the superlattice planes with

$$
S_{z}=\frac{n}{d}-\frac{m}{D}
$$

where $m$ and $n$ are integers.

As terphenylene has a threefold axis, a molecule makes a $2 \pi / 3$ turn in a pitch and the first level for the superlattice corresponds to a third order Bessel function, $J_{1}$ and $J_{2}$ terms vanish and thus the scattered intensity in the fundamental planes should be [5] :

$$
I_{0}=\left[\sum_{i} F_{i} J_{0}\left(2 \pi \mathbf{r}_{i} \cdot \mathbf{s}_{\perp}\right)\right]^{2}
$$

while the scattered intensity in the first superlattice plane is :

$$
I_{1}=\left[\sum_{i} F_{i} J_{3}\left(2 \pi \mathbf{r}_{i} \cdot \mathbf{s}_{\perp}\right)\right]^{2}
$$

where $s_{\perp}$ is the projection of the scattering vector on a plane perpendicular to $o z$,

$r_{i}$ the distance from the centre of mass and of the $i$ th atom of the molecule,

$F_{i}$ its scattering vector,

$J_{0}$ and $J_{3}$ are the zero and third order Bessel functions.

Taking into account both our geometrical conditions $\left(\theta_{\mathrm{Bragg}}<30^{\circ}\right)$ and also the decrease of the amplitude of Bessel functions with their order, we can only see reflexions corresponding to

$$
S_{z}= \pm \frac{1}{D}, \quad S_{z}= \pm\left(\frac{1}{d}-\frac{1}{D}\right)
$$

and $S_{z}= \pm \frac{1}{d}$. The plane corresponding to

$$
S_{z}=\left(\frac{1}{d}-\frac{1}{D}\right)
$$

is superimposed on the diffuse ring at $\frac{1}{4.5} \AA^{-1}$ and thus it is difficult to analyse the modulation of this ring and to give an idea concerning the order of the aliphatic chains at low temperature.

If we now consider an assembly of the same stacks of molecules forming a hexagonal packing but without correlations between the $z$ coordinates of molecules belonging to different stacks, the intensity in the fundamental zero level should be multiplied by $\delta$ functions peaked on each $h k 0$ hexagonal reciprocal point while for all the other levels we have to add the intensity diffracted by each stack.

If we only consider the rigid part of the molecule, i.e. the terphenylene and the oxygen atoms, the dependence of the intensity versus $s_{\perp}$ is easily calculated using relations (1) and (2) (Fig. 4). The features of the observed diffraction pattern (i.e. the in-plane width of the fundamental reflexion and the in-plane width and the radius of diffuse rings lying on the superlattice plane) fit nicely to the calculated intensity. We can thus conclude that the aliphatic chains do not contribute much to the intensity scattered out of the zero level : the periodicity of the molecules along the columns and the helicoidal array is mainly due to the aromatic part of the molecules while the aliphatic chains are more fluid and contribute to the diffraction in the ring at $d=4.5 \AA$. In fact, a more elaborate calculation would be needed to obtain better knowledge of the structure of the aliphatic part. From the width in the $z$ direction of the two scattering planes considered, we can deduce the correlation length for the equidistant stacking of the molecules and for the helicoidal structure. The first is too large to evaluate, the second is about $70 \AA$ at $85^{\circ} \mathrm{C}$.

The intensity of the $h k 0$ hexagonal reflexions is given by equation (1), but we have also to take into account the atoms of the pentyl chains. Without any 

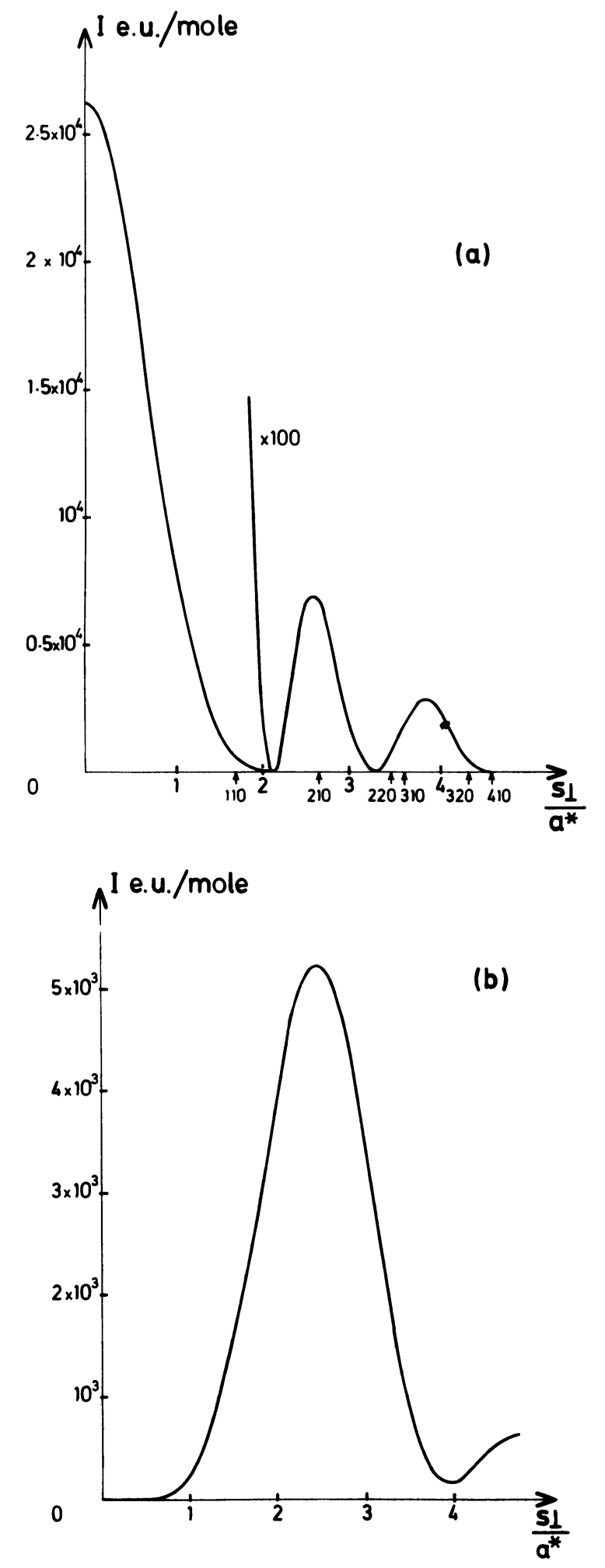

further evaluation, one can easily predict that all oscillations of the intensity plotted in figure $4 a$ have to be shrunk towards the origin of the reciprocal space as we have considered the reciprocal image of a disk smaller than the true molecule and one can see that the first order 100 reflexions must be of at least two orders of magnitude higher than the other reflexions.

The X-ray patterns of hexaheptyloxy triphenylene have the same appearance but we do not obtain good single domains and we could not establish if there is a helicoidal stacking of the core of the molecules. The distance between two parallel molecules in a stack is still defined and has the same value, $3.59 \AA$, for the two compounds, while the distance between two stacks is $21.94 \AA$ which is smaller than the diameter of the fully extended molecule; this last observation is true also for the pentyl compound. In fact, the two mesophases are compact since the calculated specific gravity is $1.11 \mathrm{~g} / \mathrm{cm}^{3}$ for the pentyl derivative and $1.01 \mathrm{~g} / \mathrm{cm}^{3}$ for the heptyl.

3. Conclusion. - The hexalcoxy triphenylene forms a mesophase, the structure of which is similar to that proposed by Chandrasekhar namely : columns of molecules forming a hexagonal packing. Nevertheless in our case the molecules have a larger rigid core and from our X-ray single domain pattern, we can distinguish a well defined periodic order of the core from a more liquid-like order in the chains. We have also described the features of the X-ray pattern related to the elastic properties of the columns of molecules.

Acknowledgments. - We are very grateful to Prof. J. Billard, Drs J. C. Dubois, Nguyen Huu Tinh and A. Zann who supplied the samples.

Fig. 4. - Calculated intensity $I$ in electron units/mole for a helix formed by the cores of the molecules versus the in-plane component of the scattering vector $s_{\perp}$ in reciprocal units of the hexagonal lattice of the $\mathrm{C}_{5}$ derivative. a) Fundamental planes, $b$ ) first level superlattice plane.

\section{References}

[1] Chandrasekhar, S., Sadashiva, B. K., Suresh, K. A., Pramana 9 (1977) 471.

[2] Spegt, P., Skoulios, A., Acta Cryst. 21 (1966) 892.

[3] Billard, J., Dubois, J. C., NguYen, N. T., ZanN, A., Nouveau Journal de Chimie 2 (1978) 535.

Dubois, J. C., Ann. Phys. 3 (1978) 131.

[4] Destrade, C., Mondon, M. C., Malthete, J., To be published : J. Physique Colloq. 40 (1979), International Conference on Liquid Crystals, Bordeaux (1978).
[5] Cochran, W., Rick, F. H. C., Vand, V., Acta Cryst. 5 (1952) 581.

[6] Doucet, J., Levelut, A. M., Lambert, M., Mol. Liq. Cryst. 24 (1973) 317.

[7] Helfrich, W., To be published : J. Physique Colloq. 40 (1979), International Conference on Liquid Crystals, Bordeaux (1978). 